\title{
Some Probability Characteristics Functions of the Solution of Stochastic Fredholm Integral Equation Contains a Known Sine Wave Function
}

\author{
Mohammad Wahdan Muflih* \\ Received 14, February, 2012 \\ Accepted 10, June , 2012
}

Amina Kassim Hussain**

\begin{abstract}
:
In this paper, some probability characteristics functions (moments, variances, convariance, and spectral density functions) are found depending upon the smallest variance of the solution of some stochastic Fredholm integral equation contains as a known function, the sine wave function.
\end{abstract}

Key words: Sine wave function, stochastic Fredholm integral equation.

\section{Introduction:}

The aim of this paper is finding some probability characteristics like moments, variances, covariance function and spectral density function, depending upon the smallest variance of the solution of the following stochastic Fredholm integral equation (SFIE),

$$
\mathbf{u}(\mathrm{w}, \mathrm{t})=\mathbf{f}(\mathrm{w}, \mathrm{t})+\int_{0}^{\infty} \mathrm{k}(\mathrm{w}, \mathrm{t}, \mathrm{s}) \mathbf{u}(\mathrm{w}, \mathrm{s}) \mathrm{ds}
$$

such that,

- $\mathrm{f}(\mathrm{w}, \mathrm{t})$ is a sine wave function (sinusoidal wave) which is a

$\mathrm{f}(\mathrm{w}, \mathrm{t})=\mathrm{A} \sin (\mathrm{wt}+\mathrm{B}), \mathrm{t}>0,0 \leq \mathrm{w} \leq 2 \pi$

where $\mathrm{A}$ is the peak deviation (amplitude) of the function from its center position, $\mathrm{w}$ is the angular frequency specify how many oscillations occur in a unit time interval (scalling by degrees per second) and B is the phase shift, [1].

- $\quad \mathrm{k}(\mathrm{w}, \mathrm{t}, \mathrm{s})$ is known stochastic kernel defined by $t>0$ and $s \in S$, where $S$ is a compact metric space.

- $\mathrm{u}(\mathrm{s}, \mathrm{t})$ is a scalar function defined for the time $t>0, s \in S$.
- stochastic process defined for $\mathrm{t}>0$ with the formula

In this paper, two cases of the phase shift B will be considered, $\mathrm{B}=\frac{\pi}{3}$ and $\mathrm{B}=\frac{\pi}{6}$ associated with two values of the amplitude A. Also, the paper consider that, the kernel $\mathrm{k}(\mathrm{w}, \mathrm{t}, \mathrm{s})$ have the following negative exponential function,

$$
\mathrm{k}(\mathrm{w}, \mathrm{t}, \mathrm{s})=\mathrm{e}^{-(\mathrm{st}+\mathrm{w})}, 0<\mathrm{s}<2 \pi, \mathrm{t}>0,0 \leq \mathrm{w} \leq \pi
$$

\footnotetext{
*Department of Mathematics-Ibn-Al-Haitham College of Education - University of Baghdad **Department of Material Engineering, College of Engineering - Al-Mustansiriya University
} 
Preliminaries

By substituting (1.2) and (1.3) into (1.1), we get

$\mathrm{u}(\mathrm{w}, \mathrm{t})=\mathrm{A} \sin (\mathrm{wt}+\mathrm{B})+\int_{0}^{\pi} \mathrm{e}^{-(\mathrm{st}+\mathrm{w})} \mathrm{u}(\mathrm{w}, \mathrm{s}) \mathrm{ds}$

To find the solution of this equation,

(A.D.M) will be used which briefly Adomain decomposition method depend on the following steps, [2],

$\mathrm{u}_{0}(\mathrm{w}, \mathrm{t})=\mathrm{f}(\mathrm{w}, \mathrm{t})=\mathrm{A} \sin (\mathrm{wt}+\mathrm{B})$

$\mathrm{u}_{0}(\mathrm{w}, \mathrm{s})=\mathrm{f}(\mathrm{w}, \mathrm{s})=\mathrm{A} \sin (\mathrm{ws}+\mathrm{B})$

$u_{m+1}(w, s)=\int_{0}^{2 \pi} e^{-(s t+w)} u_{m}(w, s) d s, m=0,1,2, \ldots$

that is to obtain the following

stochastic solution,

$\mathrm{u}(\mathrm{w}, \mathrm{t})=\mathrm{u}_{0}(\mathrm{w}, \mathrm{t})+\sum_{\mathrm{n}=1}^{\infty} \mathrm{u}_{\mathrm{n}}(\mathrm{w}, \mathrm{t})$

So that when $\mathrm{m}=0$, by (2.1b) and (2.1c),

$$
\begin{aligned}
\mathrm{u}_{1}(\mathrm{w}, \mathrm{t}) & =\mathrm{A} \int_{0}^{2 \pi} \mathrm{e}^{-(\mathrm{st}+\mathrm{w})} \sin (\mathrm{ws}+\mathrm{B}) \mathrm{ds} \\
& =\frac{\mathrm{A}}{\mathrm{t}} \mathrm{e}^{-(\mathrm{w}-\mathrm{B})} \int_{B}^{2 \pi+\mathrm{B}} \mathrm{e}^{-\mathrm{y}} \sin \mathrm{ydy}, \quad \mathrm{y}=\mathrm{ws}+\mathrm{B}
\end{aligned}
$$

or

$\mathrm{u}_{1}(\mathrm{w}, \mathrm{t})=\frac{\mathrm{A}}{2 \mathrm{t}} \mathrm{e}^{-\mathrm{w}}\left\{(\sin \mathrm{B}+\cos \mathrm{B})-\mathrm{e}^{-\pi \mathrm{t}}(\sin (2 \pi \mathrm{t}+\mathrm{B})+\cos (2 \pi \mathrm{t}+\mathrm{B}))\right\}$

and when $m=1$, by (2.1c) and (2.3),

$$
\begin{aligned}
\mathrm{u}_{2}(\mathrm{w}, \mathrm{t}) & =\int_{0}^{\pi} \mathrm{e}^{-(\mathrm{s}+\mathrm{w})} \mathrm{u}_{1}(\mathrm{~s}, \mathrm{t}) \mathrm{ds} \\
& =\frac{\mathrm{A}}{2 \mathrm{t}}\left\{(\sin \mathrm{B}+\cos \mathrm{B})-\mathrm{e}^{-\pi t}(\sin (\pi \mathrm{t}+\mathrm{B})+\cos (\pi \mathrm{t}+\mathrm{B}))\right\} \int_{0}^{\pi} \mathrm{e}^{-\mathrm{s}} \mathrm{e}^{-(\mathrm{s} t \mathrm{w})} \mathrm{ds} \\
& =\frac{\mathrm{A}}{2 \mathrm{t}} \mathrm{e}^{-\mathrm{w}}\left\{(\sin \mathrm{B}+\cos \mathrm{B})-\mathrm{e}^{-\pi t}(\sin (\pi \mathrm{t}+\mathrm{B})+\cos (\pi \mathrm{t}+\mathrm{B}))\right\} \int_{0}^{\pi} \mathrm{e}^{-(\mathrm{t} t \mathrm{t}) \mathrm{d}} \mathrm{ds}
\end{aligned}
$$

or

$\mathrm{u}_{2}(\mathrm{w}, \mathrm{t})=\frac{\mathrm{A}}{2 \mathrm{t}} \mathrm{e}^{-\mathrm{w}}\left\{(\sin \mathrm{B}+\cos \mathrm{B})-\mathrm{e}^{-\pi t}(\sin (\pi \mathrm{t}+\mathrm{B})+\cos (\pi \mathrm{t}+\mathrm{B}))\right\}\left(\frac{1-\mathrm{e}^{-\pi(t+1)}}{\mathrm{t}+1}\right)$

and when $\mathrm{m}=2$, by (2.1c) and (2.4)

$$
\begin{aligned}
& \mathrm{u}_{3}(\mathrm{w}, \mathrm{t})=\frac{\mathrm{A}}{2 \mathrm{t}} \mathrm{e}^{-\mathrm{w}}\left\{(\sin \mathrm{B}+\cos \mathrm{B})-\mathrm{e}^{-\pi \mathrm{t}}(\sin (\pi \mathrm{t}+\mathrm{B})+\cos (\pi \mathrm{t}+\mathrm{B}))\right\}\left(\frac{1-\mathrm{e}^{-\pi(t+1)}}{\mathrm{t}+1}\right) \int_{0}^{\pi} \mathrm{e}^{-(\mathrm{t}+1) \mathrm{s}} \mathrm{ds} \\
& \text { or } \\
& \mathrm{u}_{3}(\mathrm{w}, \mathrm{t})=\frac{\mathrm{A}}{2 \mathrm{t}} \mathrm{e}^{-\mathrm{w}}\left\{(\sin \mathrm{B}+\cos \mathrm{B})-\mathrm{e}^{-\pi t}(\sin (\pi \mathrm{t}+\mathrm{B})+\cos (\pi \mathrm{t}+\mathrm{B}))\right\}\left(\frac{1-\mathrm{e}^{-\pi(t+1)}}{\mathrm{t}+1}\right)^{2}
\end{aligned}
$$


By iteration for $\mathrm{m}=3,4,5, \ldots$ and adding $(2.3)-(2.5)$, we get,

$$
\begin{aligned}
\sum_{n=1}^{\infty} \mathrm{u}_{\mathrm{n}}(\mathrm{w}, \mathrm{t}) & =\frac{\mathrm{A}}{2 \mathrm{t}} \mathrm{e}^{-\mathrm{w}}\left\{(\sin \mathrm{B}+\cos \mathrm{B})-\mathrm{e}^{-\pi \mathrm{t}}(\sin (\pi \mathrm{t}+\mathrm{B})+\cos (\pi \mathrm{t}+\mathrm{B}))\right\} \sum_{\mathrm{k}=0}^{\infty}\left(\frac{1-\mathrm{e}^{-\pi(\mathrm{t}+1)}}{\mathrm{t}+1}\right) \\
& =\frac{\mathrm{A}}{2 \mathrm{t}} \mathrm{e}^{-\mathrm{w}}\left\{(\sin \mathrm{B}+\cos \mathrm{B})-\mathrm{e}^{-\pi \mathrm{t}}(\sin (\pi \mathrm{t}+\mathrm{B})+\cos (\pi \mathrm{t}+\mathrm{B}))\right\}\left(\frac{\mathrm{t}+1}{\mathrm{t}+\mathrm{e}^{-\pi(\mathrm{t}+1)}}\right)
\end{aligned}
$$

or

$$
\sum_{n=1}^{\infty} u_{n}(w, t)=A M_{(B)} e^{-w}
$$

where

$M_{(B)}=\left\{\frac{(\sin B+\cos B)-e^{-\pi t}(\sin (\pi t+B)+\cos (\pi t+B)) A}{2 t}\right\}\left(\frac{t+1}{t+e^{-\pi(t+1)}}\right)$

Finally, by substituting (2.1a), (2.6) into (2.2) which represents the stochastic solution of (2.1) we get,

$$
\mathrm{u}_{(\mathrm{B})}(\mathrm{w}, \mathrm{t})=\mathrm{A}\left[\sin (\mathrm{wt}+\mathrm{B})+\mathrm{M}_{(\mathrm{B})} \mathrm{e}^{-\mathrm{w}}\right], 0<\mathrm{w}<\pi, \mathrm{t}>0
$$

Moreover, this solution can be considered as a solution for the time $\mathrm{t}$ $\in \mathrm{T}$ such that $\{0.1,0.2,0.3, \ldots, 2.0\}$.

Moments, Variances of the

Solution:

In order to find the moments, variances of the solution (2.7), it must be that this solution is a probability density function of w. So, we equate

$$
\mathrm{A}=\frac{1}{0.975 \mathrm{M}_{(\mathrm{B})}-\frac{1}{\mathrm{t}}[\cos (\pi \mathrm{t}+\mathrm{B})-\cos \mathrm{B}]}
$$

the solution by one and find the value of the amplitude A, (i.e.), we write,

$$
\int_{0}^{\pi} u_{(B)}(w, t) d w=\int_{0}^{\pi} A\left[\sin (w t+B)+M_{(B)} e^{-w}\right] d w=1
$$

which yields to,

Hence the solution (2.7) with the following formula

$$
\mathrm{u}_{(\mathrm{B})}(\mathrm{w}, \mathrm{t})=\frac{\sin (\mathrm{wt}+\mathrm{B})+\mathrm{M}_{(\mathrm{B})} \mathrm{e}^{-\mathrm{w}}}{0.975 \mathrm{M}_{(\mathrm{B})}-\frac{1}{\mathrm{t}}[\cos (\pi \mathrm{t}+\mathrm{B})-\cos \mathrm{B}]}
$$

is a probability density function.

\section{First moment:}




$$
\begin{aligned}
\mathrm{E}_{\mathrm{u}_{(B)}}[\mathrm{w}, \mathrm{t}]= & \int_{0}^{\pi} \mathrm{w}\left[\mathrm{u}_{(\mathrm{B})}(\mathrm{w}, \mathrm{t})\right] \mathrm{dw} \\
& =\frac{\int_{0}^{\pi} \mathrm{w}\left[\sin (\mathrm{wt}+\mathrm{B})+\mathrm{M}_{(\mathrm{B})} \mathrm{e}^{-\mathrm{w}}\right] \mathrm{dw}}{0.975 \mathrm{M}_{(\mathrm{B})}-\frac{1}{\mathrm{t}}[\cos (\pi \mathrm{t}+\mathrm{B})-\cos \mathrm{B}]} \\
& =\frac{\frac{1}{\mathrm{t}^{2}}[\sin (\pi \mathrm{t}+\mathrm{B})-\sin \mathrm{B}]-\frac{\pi}{\mathrm{t}} \cos (\pi \mathrm{t}+\mathrm{B})+\left(1-\mathrm{e}^{-\pi}-\pi \mathrm{e}^{-\pi}\right) \mathrm{M}_{(\mathrm{B})}}{0.975 \mathrm{M}_{(\mathrm{B})}-\frac{1}{\mathrm{t}}[\cos (\pi \mathrm{t}+\mathrm{B})-\cos \mathrm{B}]}
\end{aligned}
$$

or

$$
\mathrm{E}_{\mathrm{u}_{(\mathrm{B})}}[\mathrm{w}, \mathrm{t}]=\frac{\frac{1}{\mathrm{t}^{2}}[\sin (\pi \mathrm{t}+\mathrm{B})-\sin \mathrm{B}]-\frac{\pi}{\mathrm{t}} \cos (\pi \mathrm{t}+\mathrm{B})+0.821 \mathrm{M}_{(\mathrm{B})}}{0.975-\frac{1}{\mathrm{t}}[\cos (\pi \mathrm{t}+\mathrm{B})-\cos \mathrm{B}]}
$$

\section{Second moment:}

$$
\begin{aligned}
\mathrm{E}_{\mathrm{u}_{(B)}}\left[\mathrm{w}^{2}, \mathrm{t}\right] & =\int_{0}^{\pi} \mathrm{w}^{2}\left[\mathrm{u}_{(\mathrm{B})}(\mathrm{w}, \mathrm{t})\right] \mathrm{dw} \\
& =\frac{\int_{0}^{\pi} \mathrm{w}^{2}\left[\sin (\mathrm{wt}+\mathrm{B})+\mathrm{M}_{(\mathrm{B})} \mathrm{e}^{-\mathrm{w}}\right] \mathrm{dw}}{0.975 \mathrm{M}_{(\mathrm{B})}-\frac{1}{\mathrm{t}}[\cos (\pi \mathrm{t}+\mathrm{B})-\cos \mathrm{B}]} \\
& =\frac{\frac{2}{\mathrm{t}^{3}}[\cos (\pi \mathrm{t}+\mathrm{B})-\cos \mathrm{B}]+\frac{2 \pi}{\mathrm{t}^{2}} \sin (\pi \mathrm{t}+\mathrm{B})-\frac{\pi^{2}}{\mathrm{t}} \cos (\pi \mathrm{t}+\mathrm{B})+\left[2-\left(\pi^{2}+2 \pi+2\right) \mathrm{e}^{-\pi}\right] \mathrm{M}_{(\mathrm{B})}}{0.975-\frac{1}{\mathrm{t}}[\cos (\pi \mathrm{t}+\mathrm{B})-\cos \mathrm{B}]}
\end{aligned}
$$

or

$$
\mathrm{E}_{\mathrm{u}_{(\mathrm{B})}}\left[\mathrm{w}^{2}, \mathrm{t}\right]=\frac{\frac{2}{\mathrm{t}^{3}}[\cos (\pi \mathrm{t}+\mathrm{B})-\cos \mathrm{B}]+\frac{2 \pi}{\mathrm{t}^{2}} \sin (\pi \mathrm{t}+\mathrm{B})-\frac{\pi^{2}}{\mathrm{t}} \cos (\pi \mathrm{t}+\mathrm{B})+1.215 \mathrm{M}_{(\mathrm{B})}}{0.975-\frac{1}{\mathrm{t}}[\cos (\pi \mathrm{t}+\mathrm{B})-\cos \mathrm{B}]}
$$

Calculations of $\operatorname{var}_{u_{(B)}}[\mathrm{w}, \mathrm{t}]$ (defined in 2.6), when $\mathrm{B}=\frac{\pi}{6}, \frac{\pi}{3}$ are tabulated for $\mathrm{t} \in \mathrm{T}=$ $\{0.1,0.2, \ldots, 2.0\}$ in tables (1), (2) which shown that the smallest variance when the phase shift $B=\frac{\pi}{6} \quad$ is $\mathrm{u}_{\left(\frac{\pi}{3}\right)}(\mathrm{w}, 0.9)=\frac{\sin \left(0.9 \mathrm{w}+\frac{\pi}{3}\right)+1.743 \mathrm{e}^{-\mathrm{w}}}{2.5484}, 0<\mathrm{w}<\pi$
$(0.05271)$ at $(\mathrm{t}=0.9)$, while the smallest variance when the phase shift $\mathrm{B}=\frac{\pi}{6}$ is $(0.02077)$ at $(\mathrm{t}=1.10)$. Therefore, the stochastic solution (3.2) can be rewritten as the following two formulas, 
$\mathrm{u}_{\left(\frac{\pi}{6}\right)}(\mathrm{w}, 1.1)=\frac{\sin \left(1.1 \mathrm{w}+\frac{\pi}{6}\right)+1.226 \mathrm{e}^{-\mathrm{w}}}{2.6188}, 0<\mathrm{w}<\pi$

(Figure (1) represent the curves of $\left.\mathrm{u}_{\left(\frac{\pi}{3}\right)}(\mathrm{w}, 0.9), \mathrm{u}_{\left(\frac{\pi}{6}\right)}(\mathrm{w}, 1.1)\right)$.

Covariance Function of of $u_{\left(\frac{\pi}{3}\right)}(\mathrm{w}, 0.9)$ is an even function
$\mathbf{u}_{(\mathbf{B})}(\mathbf{w}, \mathbf{t})$ : When $\mathrm{B}=\frac{\pi}{3}$

For any $\mathrm{s}>\mathrm{t}=0.9, \tau=\mathrm{s}-0.9$ and depends on the difference $|\mathrm{s}-0.9|=$ $|\tau|=|0.9-\mathrm{s}|$ and can be found as following, [3]

$-\tau=0.9-\mathrm{s}$, the covariance function

$\mathrm{C}(-\tau)=\mathrm{C}(0.9+\tau, 0.9)$

$=\mathrm{C}(0.9,0.9+\tau)$

$=\mathrm{C}(\tau)$

$=\mathrm{E}_{\mathrm{u}_{\left(\frac{\pi}{3}\right)}}[\mathrm{w}, 0.9]-\mathrm{E}_{\mathrm{u}_{\left(\frac{\pi}{3}\right)}}[\mathrm{w}, 0.9+\tau]-\mathrm{E}_{\mathrm{u}_{\left(\frac{\pi}{3}\right)}}[\mathrm{w}, 0.9] \mathrm{E}_{\mathrm{u}_{\left(\frac{\pi}{3}\right)}}[\mathrm{w}, 0.9+\tau]$

$=\mathrm{E}_{\mathrm{u}_{\left(\frac{\pi}{3}\right)}}\left[\mathrm{w}^{2}, 0.9\right]-\mathrm{E}_{\mathrm{u}_{\left(\frac{\pi}{3}\right)}}[\mathrm{w}, 0.9+\tau] \mathrm{E}_{\mathrm{u}_{\left(\frac{\pi}{3}\right)}}[\mathrm{w}, 0.9+\tau]-\mathrm{E}_{\mathrm{u}_{\left(\frac{\pi}{3}\right)}}\left[\mathrm{w}^{2}, 0.9\right]-\mathrm{E}_{\mathrm{u}_{\left(\frac{\pi}{3}\right)}}[\mathrm{w}, 0.9] \mathrm{E}_{\mathrm{u}_{\left(\frac{\pi}{3}\right)}}[\mathrm{w}, 0.9+\tau]$

$=\mathrm{E}_{\mathrm{u}_{\left(\frac{\pi}{3}\right)}}\left[\mathrm{w}^{2}, 0.9\right]+\mathrm{E}_{\mathrm{u}_{\left(\frac{\pi}{3}\right)}}[\mathrm{w}, 0.9] \mathrm{E}_{\mathrm{u}_{\left(\frac{\pi}{3}\right)}}[\mathrm{w}, 0.9+\tau]-\mathrm{E}_{\mathrm{u}_{\left(\frac{\pi}{3}\right)}}\left[\mathrm{w}^{2}, 0.9\right]-\mathrm{E}_{\mathrm{u}_{\left(\frac{\pi}{3}\right)}}[\mathrm{w}, 0.9] \mathrm{E}_{\mathrm{u}_{\left(\frac{\pi}{3}\right)}}[\mathrm{w}, 0.9+\tau]$

$=\mathrm{E}_{\mathrm{u}_{\left(\frac{\pi}{3}\right)}}\left[\mathrm{w}^{2}, 0.9\right]+\mathrm{E}_{\mathrm{u}_{\left(\frac{\pi}{3}\right)}}[\mathrm{w}, 0.9]\left(\mathrm{E}_{\mathrm{u}_{\left(\frac{\pi}{3}\right)}}[\mathrm{w}, 0.9+\tau]-\mathrm{E}_{\mathrm{u}_{\left(\frac{\pi}{3}\right)}}\left[\mathrm{w}^{2}, 0.9\right]\right)-\mathrm{E}_{\mathrm{u}_{\left(\frac{\pi}{3}\right)}}[\mathrm{w}, 0.9] \mathrm{E}_{\mathrm{u}_{\left(\frac{\pi}{3}\right)}}[\mathrm{w}, 0.9+\tau]$

$=\mathrm{E}_{\mathrm{u}_{\left(\frac{\pi}{3}\right)}}\left[\mathrm{w}^{2}, 0.9\right]+\mathrm{E}_{\mathrm{u}_{\left(\frac{\pi}{3}\right)}}[\mathrm{w}, 0.9] \mathrm{E}_{\mathrm{u}_{\left(\frac{\pi}{3}\right)}}[\mathrm{w}, 0.9+\tau]-\left(\mathrm{E}_{\mathrm{u}_{\left(\frac{\pi}{3}\right)}}\left[\mathrm{w}^{2}, 0.9\right]\right)^{2}-\mathrm{E}_{\mathrm{u}_{\left(\frac{\pi}{3}\right)}}[\mathrm{w}, 0.9] \mathrm{E}_{\mathrm{u}_{\left(\frac{\pi}{3}\right)}}[\mathrm{w}, 0.9+\tau]$

$=\mathrm{E}_{\mathrm{u}_{\left(\frac{\pi}{3}\right)}}\left[\mathrm{w}^{2}, 0.9\right]+\left(\mathrm{E}_{\mathrm{u}_{\left(\frac{\pi}{3}\right)}}[\mathrm{w}, 0.9]\right)^{2}$

$=\operatorname{Var}_{\left(\frac{\pi}{3}\right)}[\mathrm{w}, 0.9]$

So, by table (2) when $\mathrm{t}=0.9$,

$\mathrm{C}(-\tau)=\mathrm{C}(\tau)=0.05271, \tau>0$

When $B=\frac{\pi}{6}$

For any $\mathrm{s}>\mathrm{t}=1.1$, as previous, the covariance function of $u_{\left(\frac{\pi}{6}\right)}$ is

$\mathrm{C}(-\tau)=\mathrm{C}(\tau)=0.02077, \tau>0$

\section{Spectral Density Function}

of $\mathbf{u}_{(\mathbf{B})}(\mathbf{w}, \mathbf{t})$ :

When $B=\frac{\pi}{3}$
$\mathrm{C}(-\tau)=\mathrm{C}(\tau)=\operatorname{var}_{\mathrm{u}_{\left(\frac{\pi}{3}\right)}}[\mathrm{w}, 1.1]$

and by table (3) when $t=1.10$ 
can be found by Khinchine's formula as following, [4],

$$
\begin{aligned}
\mathrm{f}\left(\mathrm{u}_{\left(\frac{\pi}{3}\right)}, \lambda\right) & =\frac{1}{2 \pi} \int_{-\infty}^{\infty} \mathrm{C}(\tau) \mathrm{e}^{-\mathrm{i} \lambda \tau} \mathrm{d} \tau \\
& =\frac{0.5271}{2 \pi} \int_{-\infty}^{\infty}(\cos \lambda \tau-\mathrm{i} \sin \lambda \tau) \mathrm{d} \tau
\end{aligned}
$$

$\mathrm{f}\left(\mathrm{u}_{\left(\frac{\pi}{3}\right)}, \lambda\right)=0.01678 \frac{\sin [\lambda(\mathrm{s}-0.9)]}{\lambda}, \lambda \leq \mathrm{n} \pi, \mathrm{n} \in \mathrm{R}^{+} \cup\left\{0^{+}\right\}$

and for $\tau=\mathrm{s}-0.9,-\tau=0.9-\mathrm{s}$

$$
\mathrm{f}\left(\mathrm{u}_{\left(\frac{\pi}{3}\right)}, \lambda\right)=\frac{0.05271}{\pi} \int_{0}^{\mathrm{s}-0.9} \cos \lambda \tau \mathrm{d} \tau
$$

or

(Figure (2) represents the curves of for known

$\mathrm{f}\left(\mathrm{u}_{\left(\frac{\pi}{3}\right)}, \lambda\right), 0^{+} \leq \lambda \leq 2 \pi$ and $\mathrm{s}=5.9$,

10.9).

When $B=\frac{\pi}{6}$

The spectral density function of $\mathrm{u}_{\left(\frac{\pi}{6}\right)}(\mathrm{w}, 1.1)$ can be found as previously or$$
\mathrm{f}\left(\mathrm{u}_{\left(\frac{\pi}{6}\right)} ; \lambda\right)=0.00661 \frac{\sin [\lambda(\mathrm{s}-1.1)]}{\lambda}, \lambda \leq \mathrm{n} \pi, \mathrm{n} \in \mathrm{R}^{+} \cup\left\{0^{+}\right\}
$$$$
\text { and for } \tau=\mathrm{s}-1.1,-\tau=1.1-\mathrm{s}
$$$$
\mathrm{f}\left(\mathrm{u}_{\left(\frac{\pi}{6}\right)} ; \lambda\right)=\frac{0.02077}{\pi} \int_{0}^{\mathrm{s}-1.1} \cos \lambda \tau \mathrm{d} \tau
$$
following,

$$
\mathrm{f}\left(\mathrm{u}_{\left(\frac{\pi}{6}\right)} ; \lambda\right)=\frac{0.02077}{2 \pi} \int_{-\infty}^{\infty}(\cos \lambda \tau-\mathrm{i} \sin \lambda \tau) \mathrm{d} \tau
$$

Figure (3) represents the curves of $\mathrm{f}\left(\mathrm{u}_{\left(\frac{\pi}{6}\right)} ; \lambda\right), 0^{+} \leq \lambda \leq 2 \pi$ and $\mathrm{s}=6.9$, 11.1 .

\section{Conclusions :}

1- Any increase in the shift phase B followed by an increase in the value of the variance of the of the solution.

2- The speed $\mathrm{V}$ of the sine wave equation $\mathrm{V}=\frac{\mathrm{W}}{\mathrm{t}}$ when th shift phase $B=\frac{\pi}{6}(t=1.10, \quad$ variance $=0.02077)$ is less than the speed when $\mathrm{B}=\frac{\pi}{3}(\mathrm{t}=0.90$, variance $=$ 0.05271 ), that is meaning, any increase in the shift phase followed by an increase in the speed $\mathrm{V}$.

3- For future work, we recommend to find variances, spectral density functions for different values of the phase shift $\mathrm{B}\left(\mathrm{B}=0, \frac{\pi}{12}, \ldots, \frac{\pi}{2}\right)$. 


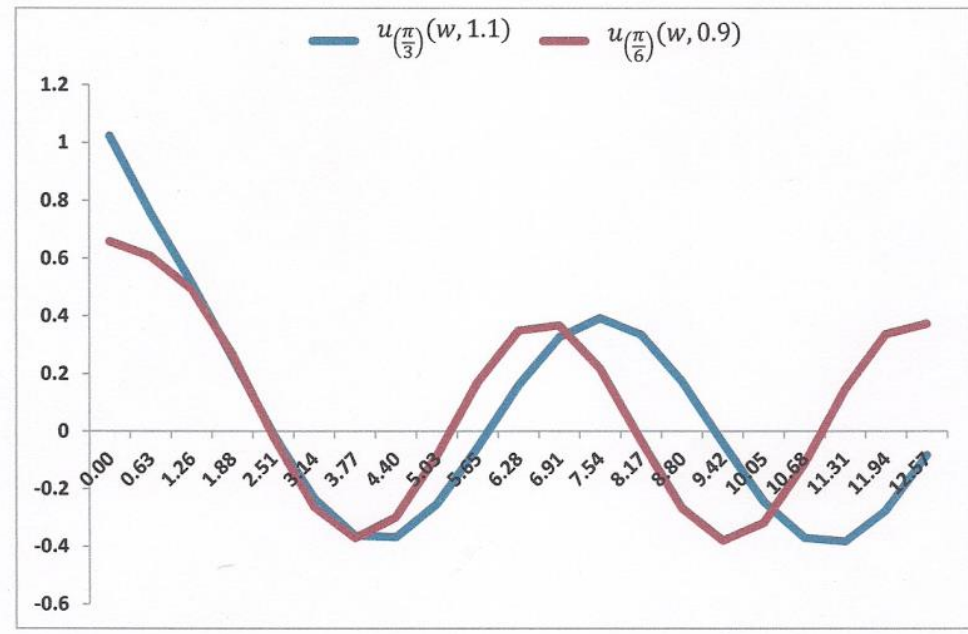

F Fig. (1)

4-

The curves of $u_{\left(\frac{\pi}{3}\right)}(w, 1.1), u_{\left(\frac{\pi}{6}\right)}(w, 0.9), 0 \leq w \leq 4 \pi$

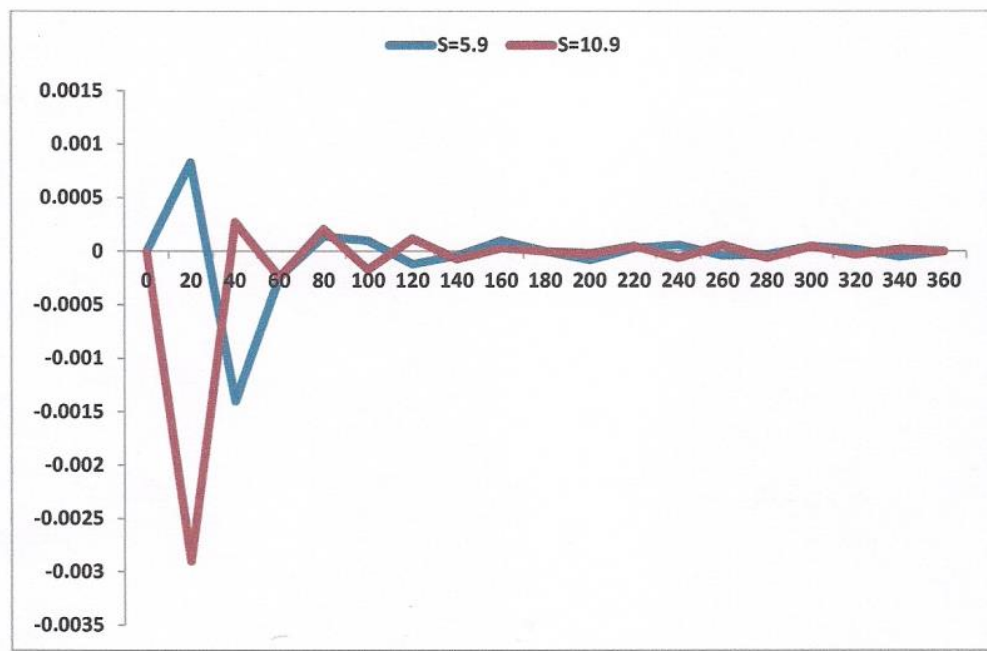

Fig. (2)

$5-$

The curves of Spectral density function ot $u_{\left(\frac{\pi}{6}\right)}(w, 0.9), 0 \leq \lambda \leq 2 \pi$

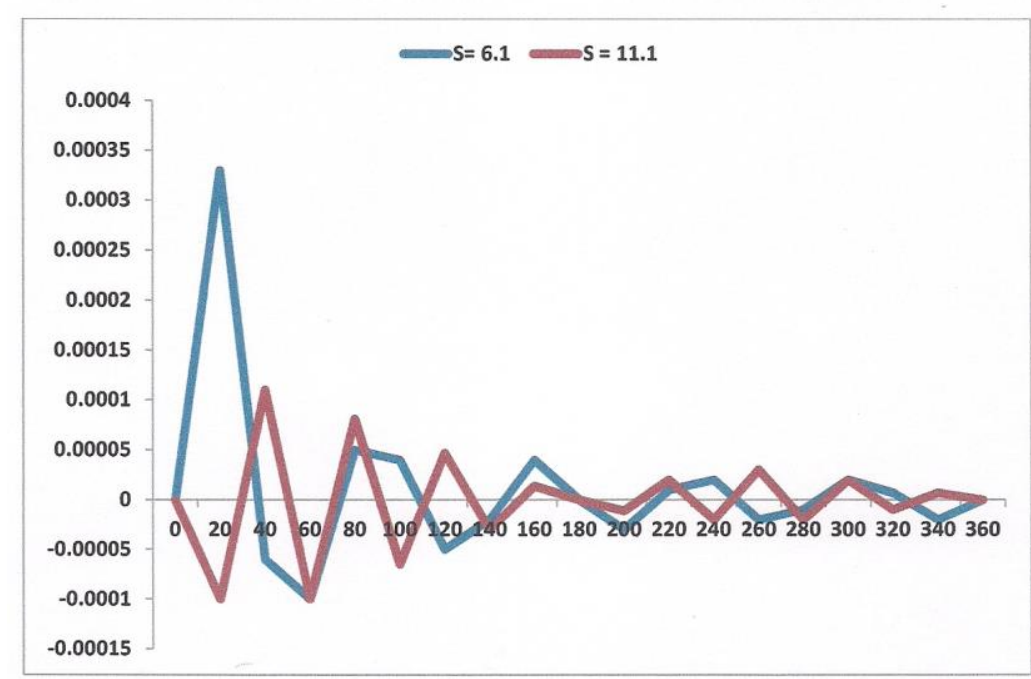

Figl Fig. (3)

6-

The curves of Spectral density function of $u_{\left(\frac{\pi}{6}\right)}(w, 1.1), 0 \leq \lambda \leq 2 \pi$ 


\section{References:}

1. Lerner, L.S., 1996, Physics for Scientistis and Engineers, John and Bartlett Pub. London, UK.

2. Vahdi, A.R. and Mokhtari, M., 2008. On Decomposition Method for System of Linear Fredholm

Integral Equations of the Second Kind, App.Math. Sci.,2(2):57-62.

3. Tanaka, H., 2002, Stochastic Process, World Scientific pub.co.pte.Ltd, Singapore.

4. Basu, A.K., 2003, Introduction to Stochastic Process, Alpha International Ltd, Pangbourne, England.

\section{بعض دوال المزايا الاحتمالية لحل معادلة فريدهولم التكاملية والعشوائية الحاوية على دالة

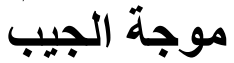

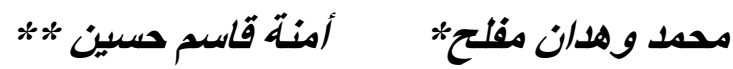

*قتم الرياضيات ـ كلية التربيةـ ابن الهيثم - جامعة بغداد

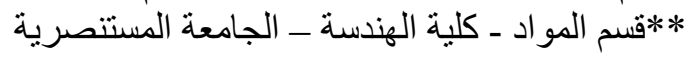

فئ هذا البحث، تم إيجاد بعض دوال المزايا الاحتمالية (العزوم، التباينات، الارتباط والكثافة الطيفية)

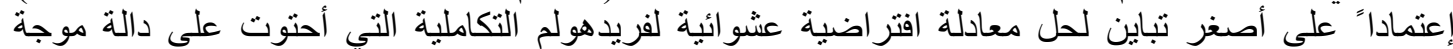

